\title{
A REMARK ON THE F. AND M. RIESZ THEOREM
}

\author{
LOUIS PIGNO AND BRENT SMITH
}

\begin{abstract}
Let $\mu$ be a measure of analytic type on the unit circle. We give a short direct proof that $\mu^{2}$ is absolutely continuous with respect to Lebesgue measure; our method also gives a convolution product version of some related several variable results.
\end{abstract}

Let $\mathbf{T}$ be the circle group, $\mathrm{Z}$ the integers and $M(\mathrm{~T})$ the complex-valued regular Borel measures on T. For $\mu \in M(\mathbf{T})$ and $n \in \mathbf{Z}$ define $\hat{\mu}(n)=\int_{\mathbf{T}} e^{-i n \theta} d \mu(\theta)$. Denote by $M_{a}(T)$ those $\mu \in M(T)$ which are absolutely continuous with respect to Lebesgue measure on $T$. We now cite the theorem of F. and M. Riesz.

THEOREM 1. Let $\mu \in M(T)$ such that $\hat{\mu}(n)=0$ for all $n<0$. Then $\mu \in M_{a}(T)$.

Several short and elegant proofs of Theorem 1 are known; see for example [1] and [2]. In this note, we prove using only the most elementary facts of harmonic analysis that the conclusion of Theorem 1 holds for convolution products. More precisely:

TheOREM 2. Let $\mu \in M(T)$ such that $\hat{\mu}(n)=0$ for all $n<0$. Then $\mu^{2} \in M_{a}(T)$.

Proof. For $\nu \in M(T)$ and $E \subset Z$ put $\|\nu\|_{B(E)}=\inf _{\rho}\{\|\rho\|: \rho \in M(T), \hat{\rho}=\hat{\nu}$ on $E$ \}. Then if $\mu \in M(T)$ with $\hat{\mu}(n)=0$ for all $n<0$ it follows that for all integers $N>0$,

$$
\|\mu\|_{B(-\infty,-N]}=0 .
$$

Let $d \tau=t d \mu$ where $t$ is a trigonometric polynomial on $\mathbf{T}$. An easy consequence of (1) is that $\lim _{N \rightarrow \infty}\|\tau\|_{B(-\infty,-N)}=0$; this implies that if $\omega \in M(T)$ and $\omega \ll \mu$ then $\lim _{N \rightarrow \infty}\|\omega\|_{B(-\infty,-N]}=0$. Let $\nu=|\mu| ;$ since $\nu \ll \mu$ we obtain $\lim _{N \rightarrow \infty}\|\nu\|_{B(-\infty,-N]}$ $=0$ and inasmuch as $\nu$ is a real measure we see that $\lim _{N \rightarrow \infty}\|\nu\|_{B(N,+\infty)}=0$. Now $\lim _{N \rightarrow \infty}\|\nu\|_{B(N,+\infty)}=0$ implies that

$$
\lim _{N \rightarrow \infty}\|\mu\|_{B[N,+\infty)}=0 .
$$

Let $\varepsilon>0$ be given. We gather from (2) that there is a measure $\mu_{\varepsilon}$ and a positive integer $N(\varepsilon)$ satisfying

$$
\left\|\mu_{\varepsilon}\right\|<\varepsilon \text { and } \hat{\mu}(n)=\hat{\mu}_{\varepsilon}(n) \text { for all } n>N(\varepsilon) .
$$

Notice that because of (1) and the second part of (3), $\mu *\left(\mu-\mu_{\varepsilon}\right)$ is a trigonometric polynomial. Inasmuch as $M_{a}(T)$ is closed, we conclude from (3) that

$$
\lim _{\ell \rightarrow 0}\left\{\mu *\left(\mu-\mu_{\varepsilon}\right)\right\}=\mu^{2} \in M_{a}(\mathbf{T}) .
$$

Received by the editors August 3, 1979.

1980 Mathematics Subject Classification. Primary 43A75. 
Comments. (a) It is trivial that Theorem 1 implies Theorem 2. Let $H^{1}(T)$ denote the measures of analytic type. A set $S \subset \mathbf{Z}$ is called a small $p$ set if whenever $\mu \in M(T)$ with supp $\hat{\mu} \subset S \Rightarrow \mu^{p} \in M_{a}(T)$; small 1 sets are called Riesz sets. Theorem 2 would imply Theorem 1 if it could be directly proved that $H^{1}(T)=$ $H^{1}(T) * H^{1}(T)$ or that every small 2 set is a Riesz set.

(b) The proof of Theorem 2 can be easily adapted to give the following three results:

(i) Let $\mathbf{R}$ be the real line and suppose $\mu \in M(\mathbf{R})$ such that $\hat{\mu}(x)=0$ for all $x<0$. Then $\mu^{2} \in M_{a}(\mathbf{R})$.

(ii) Let $S$ be a plane sector of angular opening less than $\pi$ radians. If $\mu \in M\left(T^{2}\right)$ and supp $\hat{\mu} \subset S$ then $\mu^{2} \in M_{a}\left(T^{2}\right)$.

(iii) For $n$ a natural number put $Q_{n}=\left\{\left(m_{1}, \ldots, m_{n}\right): m_{i}>0, m_{i} \in \mathbf{Z}\right\}$. If $\mu \in M\left(T^{n}\right)$ and supp $\hat{\mu} \subset Q_{n}$ then $\mu^{2} \in M_{a}\left(T^{n}\right)$.

(c) The argument in Theorem 2 that formula (1) implies formula (2) is due essentially to A. Rajchman.

\section{REFERENCES}

1. H. Helson, On a theorem of F. and M. Riesz, Colloq. Math. 3 (1955), 113-117.

2. B. K. Oksendal, A short proof of the F. and M. Riesz Theorem, Proc. Amer. Math. Soc. 30 (1971), 204.

Department of Mathematics, Kansas State University, Manhattan, Kansas 66506

DePartiment of Mathematics, Illinois State University, Normal, IllnNois 61761 